Article

\title{
Analysis of the State-Dependent Queueing Model and Its Application to Battery Swapping and Charging Stations
}

\author{
Doo Il Choi ${ }^{1}$ and Dae-Eun Lim ${ }^{2, *}$ (1) \\ 1 Department of Applied Mathematics, Halla University, 28 Halla University-gil, Wonju-si, \\ Gangwon-do 26404, Korea; dichoi@halla.ac.kr \\ 2 Department of Industrial Engineering, Kangwon National University, 1 Kangwondaehak-gil, Chuncheon-si, \\ Gangwon-do 24341, Korea \\ * Correspondence: del@kangwon.ac.kr; Tel.: +82-33-250-6282
}

Received: 21 January 2020; Accepted: 10 March 2020; Published: 17 March 2020

\begin{abstract}
This study analyzes the performance of a queue length-dependent overload control policy using a leaky bucket (LB) scheme. This queueing model is applied to the operation of a battery swapping and charging station for electric vehicles (EVs). In addition to the LB scheme, we propose two congestion control policies based on EV queue length thresholds. With these policies, the model determines both EV-arrival and battery-supply intervals, and these depend on the number of EVs waiting in the queue. The queue length distributions, including those at arbitrary epochs, are derived using embedded Markov chain and supplementary variable methods. Performance measures such as blocking probability and mean waiting time are investigated using numerical examples. We study the characteristics of the system using numerical examples and use a cost analysis to investigate situations in which the application of each congestion control policy is advantageous.
\end{abstract}

Keywords: congestion control; leaky bucket; finite queueing model; electric vehicles; battery swapping and charging station; cost analysis

\section{Introduction}

Electric vehicles (EVs) have gained attention as alternative vehicles which can reduce carbon emissions. However, there are several unsolved problems to be addressed, one of which is the 'range anxiety' problem [1]. Accurate prediction of range is complicated because it depends on various factors; thus, EV-users can often feel anxious about depleting their battery before they reach their destinations [2]. As a possible solution, the deployment of battery swapping and charging stations (BSCSs) (as in Figure 1) has been proposed [3] and is being actively reviewed for commercial use [4,5]. This study deals with the economics of operating a BSCS and quality of service (Qos) is also considered. At a BSCS, the depleted batteries of EVs can be exchanged with fully charged ones, or the depleted batteries can be charged at charging stations. One advantage of the BSCS is that waiting times for EV drivers relatively short because batteries can be exchanged within several minutes [6].

A leaky bucket (LB) scheme has been applied to communication systems and was once called "the most promising policy for controlling or preventing congestion in networks" [7]. In addition to telecommunication systems, this scheme has also been applied to other types of recently-deployed systems, including the BSCS $[6,8]$. To examine the operation of this scheme, we will first discuss the example of BSCSs for EVs in which a method of battery exchange is employed. When an EV in Figure 1 comes to a BSCS, its battery is replaced with a charged battery or, if there is no charged battery available, the vehicle must wait until a charged battery is supplied. The LB scheme, as applied to the BSCS, includes two paired queues; one is an EV queue and the other is a battery queue. The battery of 
an EV in the EV queue should be replaced with a charged battery, and they can be replaced only if a battery exists in the battery queue. A detailed explanation is given and three cases can be considered for arrival of an EV at the queue: (i) If there is no EV in the EV queue (i.e., the size of the EV queue is 0) and one or more batteries exist in the battery queue, then the battery of this EV is replaced and it is assumed to leave the BSCS immediately, (ii) when the EV arrives, it must wait until the next battery is supplied if the size of the EV queue is 0 and there is no battery in the battery queue. Subsequently, the battery is exchanged as soon as a charged battery is supplied. The battery is supplied according to a predetermined time interval, i.e., a battery-supply interval, and (iii) when the EV arrives, it must wait during the battery replacement for other EVs that arrived earlier in the EV queue. When all the EVs that have arrived earlier have left the charging station and, if there is a battery available at the turn of the corresponding EV, the battery of this EV is replaced immediately. If no battery is present, the EV driver must wait until a battery is supplied. In other words, an EV and a battery have a 1:1 relationship, and if there is no battery, the EV cannot leave the charging station. Moreover, if it is assumed that the sizes of both the EV queue and the battery queue are limited, the batteries and EVs are all blocked when the corresponding queues are full.

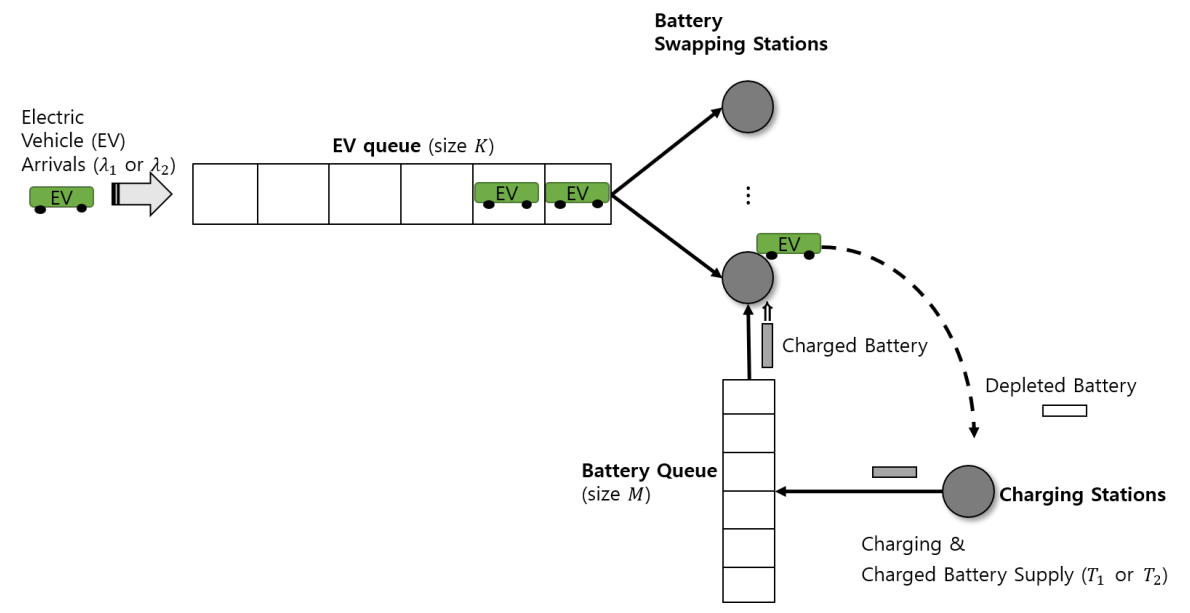

Figure 1. Model for the battery swapping and charging station for electric vehicles.

Many studies on the LB scheme applied to telecommunication systems have been conducted to mitigate the congestion in a data packet queue. A packet (voice, video, etc.) to be transmitted corresponds to an EV requesting a battery exchange in the above-mentioned BSCS, and a token corresponds to a fully charged battery. As an example, wireless sensor networks (WSNs) are frequently used in many areas for monitoring tasks such as environmental sensing, forest-fire surveillance, danger detection, and military operations [9]. An energy-harvesting WSN constructed by applying the LB scheme has been described in De Cuypere et al. [10]. Congestion control in WSNs has attracted interest from many researchers, and various methods have been proposed [11]. This is because a high level of congestion causes loss or delay (long waiting time) of the data to be transmitted, thereby deteriorating the performance of WSNs severely. Data awaiting transmission in the WSN will require a certain amount of electrical energy for transmission, and the electrical energy corresponds to a token or a fully-charged battery in the BSCS model. In the LB scheme applied to communication systems, the token generation interval can be adjusted to avoid the degradation of QoS: The interval is shortened, meaning more tokens are generated in a unit time. While generating tokens may rapidly may require more resources, it helps to shorten the waiting time of packets and reduces the number of blocked packets. The BSCS service facility should also consider the appropriate customer service waiting time to guarantee QoS. As for the BSCS, waiting time can be reduced by adjusting the supply interval for charged batteries. A short battery-supply interval requires holding more batteries (thus, a larger investment), and operational cost increases. Therefore, an appropriate point of compromise is necessary to balance operational cost and customer dissatisfaction by long waiting time. 
The contribution of this paper is two-fold. First, we propose a novel LB scheme and follow with mathematical analysis. Our LB scheme can be applied to alleviate the congestion in any LB scheme systems and is not limited to BSCSs. Second, we investigate the economics of operation numerically for a BSCS by applying our LB scheme, and then managerial implications arising from various volatile conditions and several types of costs.

The remainder of this paper is organized as follows: Section 2 reviews related literature. Section 3 introduces the novel control policies, and it is followed by mathematical modeling and analysis. In Section 4, numerical examples and the characteristics of the proposed congestion-control policy are presented. Section 5 concludes the paper.

\section{Literature Review}

In a recent study, Tan et al. [6] divided the study of BSCSs into two streams: One involves planning and design and the other considers the scheduling and operation of a BSCS. The planning and design issues are related to the location or the network of BSCSs and the capacity of the BSCS. Jamian et al. [12] tried to determine placement and size for battery switching stations. Research on the scheduling and operations directed to finding the optimal charging strategy to minimize or maximize objectives. As a scheduling and inventory management problem, Raviv [13] considered the business model of a company and formulated it as the battery switching station scheduling problem. For a more detailed literature review, refer to Tan et al. [6] and references therein. Recently, the application of robotic mobile fulfillment systems to the operation of BSCSs has been presented [14]. In this case, batteries are stored on movable shelves and movable robots are deployed to transport the shelves.

Tan et al. [6] pointed out that "Despite the above literature, there has been little work focusing on the QoS analysis of BSCSs". They analyzed a BSCS using stochastic models and a mixed queueing network, to facilitate effective and practical capacity planning. Their capacity planning includes four parameters: The number of parking spaces, the number of swapping islands (servers for swapping), the number of chargers, and the number of batteries. Tan et al. [6] and this contribution deal with the same subject - the QoS in BSCSs. To compare our model with that of Tan et al. [6], we note that both consider the number of parking spaces and, additionally, we consider the size of the buffer for charged batteries. The number of chargers and the number of batteries are related to the battery-supply intervals in our model. We does not include the number of swapping islands and assume that this is sufficient. One obvious difference is that we are focusing on a congestion control policy, and cost analysis is presented.

There are various approaches for mitigating congestion in the system and these are triggered by total workload in the system or the system size (queue length), etc. Among them, we adopt a queue length-dependent congestion control method. There are one or two more thresholds: If the queue length becomes larger than one of thresholds, service or arrival rate is regulated. The $M / G / 1$ queueing model with variable service rates with one threshold was first introduced by Nishimura and Jiang [15], and many follow-up studies have been presented: These includes a batch arrival model [16] in which arrival distribution is generalized by assuming a Markovian arrival process (MAP) [17], batch MAP (BMAP) [18,19], discrete-time MAP (DMAP) [20], etc. While there is research designed to adapt service rates, the need for more research directions on controlling arrival rates has also been raised[19,21,22]. In addition, two types of rates, service and arrival, are also regulated in a single model [23].

In other work on the LB model, Lee and Un [24], Choi and Choi [25], and Choi et al. [26] analyzed LB schemes with dynamic token generation intervals. Lee and Un [24] analyzed an LB scheme with an on-off data source in which the token generation interval during the on-period is smaller than that during the off-period. They analyzed the performance of this LB scheme using the fluid-flow method. The dynamic rate LB scheme, in which the token generation interval alternates according to buffer occupancy, was analyzed by Choi and Lee [27] and Choi [28]. Previous models control only token generation rates (or intervals), but our modified LB scheme regulates two intervals and is described in detail in Section 3. 


\section{Mathematical Model Analysis and Application}

\subsection{Model Description}

First, we illustrate our model in Figure 1 and the mathematical terms will be explained in detail. EVs are assumed to arrive at an EV queue with finite capacity $K$, according to the Poisson distribution of the parameter $\lambda_{1}$ (or $\lambda_{2}$ ). The arriving EVs are held in the EV queue if no charged batteries are available. Batteries enter the system with constant time intervals, denoted by $T_{1}$ (or $T_{2}$ ), and a combination of one EV and a single charged battery can leave the system together. Constant time intervals are assumed for $T_{1}$ and $T_{2}$, but this assumption is apparently realistic at an actual BSCS and a constant interval represents a goal in station operation. This value is used as an index that intuitively expresses the performance of a complex system. In manufacturing lines, this index is referred to as "takt time", and it assists in evaluating the performance of certain processes and in avoiding complicated methodologies. The takt time is related to the size of the BSCS. As the number of charging slots increases, the battery-supply interval decreases. Therefore, the capacity of a BSCS can be simplified and considered as the battery-supply interval. The battery queue has a finite capacity $M$ and a battery arriving when the battery queue is already full is assumed to be blocked. Blocking means that a fully-charged battery cannot enter the battery queue and should be kept in a charging station until the battery queue has a vacant slot. It seems that this assumption is not realistic, but it can be justified by assuming sufficiently large $M$. The $M$ size can refer to a physical space but can also be used to refer the number of EVs that can be served (i.e., processing capability). The congestion control policies presented in this paper are based on thresholds for the number of EVs waiting in the EV queue. Two thresholds are assumed and are denoted by $L_{1}$ and $L_{2}\left(L_{1} \leq L_{2} \leq K\right)$.

As discussed earlier, two intervals are adjusted to mitigate congestion and to prevent arriving EVs from being blocked, and this depends on the EV queue size. The first interval is the battery-supply interval, and shortening this should correspond to consuming the capability of a BSCS. We assume that a BSCS does not usually use its full of capability, and it can increase its processing capability when congestion is observed. The other intervals are those seen between the arrivals of EVs (interarrivals of EVs), and controlling arrivals would seem to be impractical. However, this could mean rerouting input streams in advance by notifying EV drivers when waiting times are long; and example of controlling input rates in semiconductor fabrication can be found in Banik [19]. In a case in which the status of the entire system can be monitored, such as in a manufacturing line, rerouting or bypassing can easily be applied by distributing jobs from a particular machine to other machines. In a road network, the bypassing application is hindered by the difficulty in monitoring the entire system. However, we assume that this can be overcome by using the internet to share information regarding the status of BSCSs and whether they are congested.

We now introduce two new congestion control policies. There are two thresholds $L_{1}$ and $L_{2}$ applied to the number of waiting EVs, and the model dynamically controls two intervals related to the thresholds: (i) Battery-supply intervals, and (ii) EV-arrival intervals. In the first combination, EV-arrival intervals are shortened first, and then shorter battery-supply intervals are implemented. We refer to this as arrival first (AF) policy. In the second combination, the order is reversed: The battery-supply interval is adjusted first, and then the interarrival times of EVs are adjusted. This is referred to as the supply first (SF) policy.

The description for the congestion control policies are given in detail in Sections 3.2 and 3.3. We start our analysis with the AF model and then follow the SF model by modifying the definition and probabilities in the analysis of the AF model. The proposed models are analyzed using the embedded Markov chain method and the supplementary variable method. The instants at which batteries enter the system are regarded as embedding points. Then, the stationary system distribution at the embedding points is derived using the embedded Markov chain method. The stationary probability distribution of the system state at arbitrary epochs is obtained using the supplementary variable method. 


\subsection{Analysis of an Arrival First Model}

To obtain the stationary probability distribution of the system state, we first use the embedded Markov chain method by considering the battery-supply instant as an embedded epoch. The EV and the battery queues are observed immediately after the battery-supply epoch. Here, $B_{n}\left(T_{n}\right)$ is the number of EVs (batteries) in their designated buffers immediately after the $n$-th battery-supply instant. Then the state of the EV and battery queues denoted by $N_{n}$ can be expressed as follows:

$$
N_{n} \triangleq B_{n}+M-T_{n}
$$

Note that the arriving EVs wait in the EV queue only if there is no battery. Moreover, the arriving charged battery waits in the battery queue only if there are no EVs; otherwise, if there are EVs present on the battery arrival epoch, the arriving charged battery leaves the system with an EV immediately. If there are $i(0 \leq i \leq M)$ batteries in the battery queue (necessarily, $\left.B_{n}=0\right)$, then $N_{n}=M-i$. Moreover, if there are $i(0 \leq i<K)$ EVs in the EV queue (likewise, $\left.T_{n}=0\right)$, then $N_{n}=M+i$. Specifically, $N_{n}=M$ when there are no EVs and no batteries. The condition $0<N_{n}<M$ means there are no EVs, but there are $M-N_{n}$ batteries. Finally, $M<N_{n}<K$ indicates that there are $\left(N_{n}-M\right)$ EVs, but no batteries. In that case, the process $\left\{N_{n}, n \geq 0\right\}$ is a Markov chain with finite state space $\{0,1, \cdots, M+K-1\}$. To obtain the system state distribution at the battery-supply instants, the number of EV arrivals during the battery-supply intervals (denoted by $T_{1}$ or $T_{2}$ ) is required. We examine the operation of the system at two successive battery-supply epochs and the number of EV arrivals during the interval. Here, $i$ denote the number of EVs in the EV queue immediately after a battery-supply event $(0 \leq i<M+K)$.

- If $0 \leq i<L_{1}$, an EV-arrival rate is $\lambda_{1}$, and a battery-supply interval is $T_{1}$. Note that arrival rates and supply intervals are adjusted according to the number of EVs in the EV queue immediately after a battery-supply event.

- If $L_{1} \leq i<L_{2}$, an EV-arrival rate is set to $\lambda_{2}$, and a battery-supply interval is $T_{1}$. Note that the "AF" policy is assumed.

- If $L_{2} \leq i<M+K$, an EV-arrival rate is $\lambda_{2}$, and a battery-supply interval is set to $T_{2}$.

Next, we introduce the following probabilities regarding the number of EV arrivals during the battery-supply intervals:

$$
\begin{aligned}
& a_{n}^{r}= \operatorname{Pr}\left\{n \mathrm{EV}(\mathrm{s}) \text { arrived by Poisson process with rate } \lambda_{r}\right. \\
&\text { during the battery-supply interval } \left.T_{r}\right\}
\end{aligned}
$$

let,

$$
\begin{aligned}
& b_{n}= \operatorname{Pr}\left\{n \mathrm{EV}(\mathrm{s}) \text { arrived by Poisson process with rate } \lambda_{2}\right. \\
&\text { during the battery-supply interval } \left.T_{1}\right\} \\
&=\frac{\left(\lambda_{2} T_{1}\right)^{n}}{n !} e^{-\lambda_{2} T_{1}}, \\
& \bar{b}_{n}=\sum_{k=n}^{\infty} b_{k} .
\end{aligned}
$$


The variables $a_{n}^{1}, b_{n}$ and $a_{n}^{2}$ are applied to the first, second, and third bullet points, respectively. Subsequently, the one-step transition probability matrix $\bar{Q}$ for the Markov chain $\left\{N_{n}, n \geq 0\right\}$ is given by

$$
\bar{Q}=\left(\begin{array}{cccccccccccccc}
a_{0}^{1}+a_{1}^{1} & a_{2}^{1} & a_{3}^{1} & \ldots & a_{M+L_{1}}^{1} & a_{M+L_{1}+1}^{1} & a_{M+L_{1}+2}^{1} & \ldots & a_{M+L_{2}}^{1} & a_{M+L_{2}+1}^{1} & a_{M+L_{2}+2}^{1} & \ldots & a_{M+K-1}^{1} & \bar{a}_{M+K}^{1} \\
a_{0}^{1} & a_{1}^{1} & a_{2}^{1} & \ldots & a_{M+L_{1}-1}^{1} & a_{M+L_{1}}^{1} & a_{M+L_{1}+l}^{1} & \ldots & a_{M+L_{2}-1}^{1} & a_{M+L_{2}}^{1} & a_{M+L_{2}+1}^{1} & \ldots & a_{M+K-2}^{1} \\
0 & a_{0}^{1} & a_{1}^{1} & \ldots & a_{M+L_{1}-2}^{1} & a_{M+L_{1}-1}^{1} & a_{M+L_{1}}^{1} & \ldots & a_{M+L_{2}-2}^{1} & a_{M+L_{2}-1}^{1} & a_{M+L_{2}}^{1} & \ldots & a_{M+K-3}^{1} & \bar{a}_{M+K-2}^{1} \\
\vdots & \vdots & \vdots & \ddots & \vdots & \vdots & \vdots & \ddots & \vdots & \vdots & \vdots & \ddots & \vdots & \vdots \\
0 & 0 & 0 & \ldots & a_{1}^{1} & a_{2}^{1} & a_{3}^{1} & \ldots & a_{L_{2}-L_{1}+1}^{1} & a_{L_{2}-L_{1}+2}^{1} & a_{L_{2}-L_{1}+3}^{1} & \ldots & a_{K-L_{1}}^{1} & \bar{a}_{K-L_{1}+1}^{1} \\
0 & 0 & 0 & \ldots & b_{0} & b_{1} & b_{2} & \ldots & b_{L_{2}-L_{1}} & b_{L_{2}-L_{1}+1} & b_{L_{2}-L_{1}+2} & \ldots & b_{K-L_{1}-1} & \bar{b}_{K-L_{1}} \\
0 & 0 & 0 & \ldots & 0 & b_{0} & b_{1} & \ldots & b_{L_{2}-L_{1}-1} & b_{L_{2}-L_{1}} & b_{L_{2}-L_{1}+1} & \ldots & b_{K-L_{1}-2} & \bar{b}_{K-L_{1}-1} \\
\vdots & \vdots & \vdots & \ddots & \vdots & \vdots & \vdots & \ddots & \vdots & \vdots & \vdots & \ddots & \vdots & \vdots \\
0 & 0 & 0 & \ldots & 0 & 0 & 0 & \ldots & b_{1} & b_{2} & b_{3} & \ldots & b_{K-L_{2}} & \bar{b}_{K-L_{2}+1} \\
0 & 0 & 0 & \ldots & 0 & 0 & 0 & \ldots & a_{0}^{2} & a_{1}^{2} & a_{2}^{2} & \ldots & a_{K-L_{2}-1}^{2} & \bar{a}_{K-L_{2}}^{2} \\
0 & 0 & 0 & \ldots & 0 & 0 & 0 & \ldots & 0 & a_{0}^{2} & a_{1}^{2} & \ldots & a_{K-L_{2}-2}^{2} \bar{a}_{K-L_{2}-1}^{2} \\
\vdots & \vdots & \vdots & \ddots & \vdots & \vdots & \vdots & \ddots & \vdots & \vdots & \vdots & \ddots & \vdots & \vdots \\
0 & 0 & 0 & \ldots & 0 & 0 & 0 & \ldots & 0 & 0 & 0 & \ldots & a_{1}^{2} & \bar{a}_{2}^{2} \\
0 & 0 & 0 & \ldots & 0 & 0 & 0 & \ldots & 0 & 0 & 0 & \ldots & a_{0}^{2} & \bar{a}_{1}^{2}
\end{array}\right) .
$$

We define the stationary probability distribution of the Markov chain $\left\{N_{n}, n \geq 0\right\}$ as

$$
x_{k}=\lim _{n \rightarrow \infty} \operatorname{Pr}\left\{N_{n}=k\right\}, 0 \leq k \leq M+K-1,
$$

and its vector form is given by

$$
\mathbf{x}=\left(x_{0}, x_{1}, \cdots, x_{M+K-1}\right) .
$$

Subsequently, the stationary probability distribution $\mathbf{x}$ of the Markov chain $\left\{N_{n}, n \geq 0\right\}$ is obtained by solving the equations

$$
\mathbf{x} \bar{Q}=\mathbf{x}, \mathbf{x e}=1 .
$$

We now derive the system state distribution at arbitrary time. Let $N(t)$ indicate the system state $\left(N_{n}\right)$ at time $t$. The the stationary probabilities are defined as

$$
y_{n}=\lim _{t \rightarrow \infty}\{N(t)=n\}, 0 \leq n \leq M+K .
$$

Subsequently, $y_{n}$ can be derived using the supplementary variable method. Let $\tilde{T}$ and $\hat{T}$ be the elapsed and remaining token entry intervals at arbitrary time, respectively. Moreover, we introduce the notation

$$
\xi(t)=\left\{\begin{array}{ll}
1, & \text { if a battery enters by interval } T_{1} \text { at time } t \\
2, & \text { if a battery enters by interval } T_{2} \text { at time } t
\end{array} .\right.
$$

Furthermore, we define the joint probability distribution of the system state and the remaining battery entry interval as follows:

$$
\alpha_{r}(n, x) d x=\lim _{t \rightarrow \infty} \operatorname{Pr}\{N(t)=n, \xi(t)=r, x<\hat{T} \leq x+d x\}, r=1,2 .
$$

Let $\alpha_{r}^{*}(n, s)$ be the Laplace transform of $\alpha_{r}(n, x)$.

$$
\alpha_{r}^{*}(n, s)=\int_{0}^{\infty} e^{-s x} \alpha_{r}(n, x) d x .
$$


To derive $\alpha_{r}^{*}(n, s)(r=1,2)$, we define the joint probability $\beta_{r}(n, x) d x$ as

$$
\begin{aligned}
& \beta_{r}(n, x) d x=\lim _{t \rightarrow \infty} \operatorname{Pr}\left\{n \text { EV arrivals by } \lambda_{r} \text { during } \tilde{T}, \xi(t)=r, x<\hat{T} \leq x+d x\right\}, \\
& \beta_{b}(n, x) d x=\lim _{t \rightarrow \infty} \operatorname{Pr}\left\{n \text { EV arrivals by } \lambda_{2} \text { during } \tilde{T}, \xi(t)=1, x<\hat{T} \leq x+d x\right\} .
\end{aligned}
$$

We also define the Laplace transform $\beta_{r}^{*}(n, s)$ of $\beta_{r}(n, x)$ as

$$
\beta_{r}^{*}(n, s)=\int_{0}^{\infty} e^{-s x} \beta_{r}(n, x) d x .
$$

Thus, $\alpha_{r}^{*}(n, s)(r=1,2)$ satisfies the following equations: For $0 \leq n<M+K$,

$$
\begin{aligned}
\alpha_{1}^{*}(n, s)= & \frac{T_{1}}{E}\left[\sum_{k=0}^{\min \left\{n, M+L_{1}-1\right\}} x_{k} \beta_{1}^{*}(n-k, s)\right. \\
& \left.+\sum_{\min \left\{n, M+L_{2}-1\right\}} x_{k} \beta_{b}^{*}(n-k, s) \mathbf{1}_{\left\{n \geq M+L_{1}\right\}}\right], \\
\alpha_{2}^{*}(n, s)= & \frac{T_{2}}{E} \sum_{k=M+L_{2}}^{n} x_{k} \beta_{2}^{*}(n-k, s) \mathbf{1}_{\left\{n \geq M+L_{2}\right\}} .
\end{aligned}
$$

where $E \triangleq \sum_{k=0}^{M+L_{2}-1} x_{k} T_{1}+\sum_{k=M+L_{2}}^{M+K-1} x_{k} T_{2}$ is the battery entry interval. $\mathbf{1}_{\text {\{condition\} }}$ is equal to 1 (or 0 ) if the "condition" is true (or false). In an analogous manner to Lee [29] (see Appendix in [29]), $\beta_{r}^{*}(n, s)$ is given as follows:

$$
\begin{aligned}
& \beta_{r}^{*}(n, s)=\frac{1}{T_{r}}\left[\sum_{k=0}^{n} a_{k}^{r} R_{n-k}^{r}(s)-e^{-s T_{r}} R_{n}^{r}(s)\right], r=1,2, \\
& \beta_{b}^{*}(n, s)=\frac{1}{T_{1}}\left[\sum_{k=0}^{n} b_{k} R_{n-k}^{2}(s)-e^{-s T_{1}} R_{n}^{2}(s)\right],
\end{aligned}
$$

where $R_{n}^{r}(s)=\left(s-\lambda_{r}\right)^{-1}\left\{-\lambda_{r}\left(s-\lambda_{r}\right)^{-1}\right\}^{n}$. Finally, substituting $\beta_{r}^{*}(n, s)(r=1,2, b)$ into the above equations (15) and considering $s=0$, we obtain the system state distribution at an arbitrary instant, denoted by $y_{n}\left(=\alpha_{1}^{*}(n, 0)+\alpha_{2}^{*}(n, 0)\right)$, as follows: For $0 \leq n<M+K$,

$$
\begin{aligned}
y_{n}=\frac{1}{E}[ & \frac{1}{\lambda_{1}} \sum_{k=0}^{\min \left\{n, M+L_{1}-1\right\}} x_{k}\left(1-\sum_{m=0}^{n-k} a_{m}^{1}\right) \\
& +\frac{1}{\lambda_{2}} \sum_{k=M+L_{1}}^{\min \left\{n, M+L_{2}-1\right\}} x_{k}\left(1-\sum_{m=0}^{n-k} b_{m}\right) \mathbf{1}_{\left\{n \geq M+L_{1}\right\}} \\
& \left.+\frac{1}{\lambda_{2}} \sum_{k=M+L_{2}}^{n} x_{k}\left(1-\sum_{m=0}^{n-k} a_{m}^{2}\right) \mathbf{1}_{\left\{n \geq M+L_{2}\right\}}\right]
\end{aligned}
$$

$y_{M+K}=1-\sum_{n=0}^{M+K-1} y_{n}$. Finally, the performance measures can be obtained using the stationary system state distribution $y_{n}$. The EV blocking probability $\left(P_{\text {Block }}\right)$, which is the probability that an arriving EV finds the EV queue to be full, is given by

$$
P_{\text {Block }}=y_{M+K} \text {. }
$$


Therefore, the mean length of the EV queue $\left(L_{E V}\right)$ and the mean waiting time $\left(W_{E V}\right)$ of a EV are calculated as follows:

$$
\begin{gathered}
L_{E V}=\sum_{i=M}^{M+K}(i-M) y_{i}, \\
W_{E V}=\frac{L_{E V}}{\left(\lambda_{1}+\lambda_{2}\right)\left(1-P_{\text {Block }}\right)} .
\end{gathered}
$$

Moreover, the mean length of the battery queue $\left(L_{B}\right)$ is given by

$$
L_{B}=\sum_{i=0}^{M}(M-i) y_{i}
$$

\subsection{Analysis of a Supply First Model}

In this section, we analyze the "SF" model. Initially, the system has two parameters, the $\lambda_{1}$ (arrival rate) and $T_{1}$ (battery entry interval) seen in the case of the "AF" model. If the EV queue length at the instance of a battery entry exceeds the threshold $L_{1}$, the battery entry interval is changed from $T_{1}$ to $T_{2}$. We note that the EV queue size then decreases during the time of the battery entry intervals. However, if the EV queue length exceeds the upper threshold $L_{2}\left(\geq L_{1}\right)$, the arrivals follow a Poisson process with rate $\lambda_{2}$. Otherwise, the arrivals follow a Poisson process with rate $\lambda_{1}$. First, we modify Equation (3) with the definition of $b_{n}$ in Section 3.2 as follows:

$$
\begin{aligned}
& b_{n}= \operatorname{Pr}\left\{n \mathrm{EV}(\mathrm{s}) \text { arrived by Poisson process with rate } \lambda_{1}\right. \\
&\text { during the battry supply interval } \left.T_{2}\right\} \\
&=\frac{\left(\lambda_{1} T_{2}\right)^{n}}{n !} e^{-\lambda_{1} T_{2}} \\
& \bar{b}_{n}=\sum_{k=n}^{\infty} b_{k} .
\end{aligned}
$$

Note that the definitions of the probabilities $a_{n}^{r}$ and $\bar{a}_{n}^{r}$ are the same as Equation (2). Thus, by applying the same method in Section 3.2, we obtain the stationary system state distribution $y_{n}$ : For $0 \leq n<M+K$,

$$
\begin{aligned}
y_{n}=\frac{1}{E}[ & \frac{1}{\lambda_{1}} \sum_{k=0}^{\min \left\{n, M+L_{1}-1\right\}} x_{k}\left(1-\sum_{m=0}^{n-k} a_{m}^{1}\right) \\
& +\frac{1}{\lambda_{1}} \sum_{k=M+L_{1}}^{\min \left\{n, M+L_{2}-1\right\}} x_{k}\left(1-\sum_{m=0}^{n-k} b_{m}\right) \mathbf{1}_{\left\{n \geq M+L_{1}\right\}} \\
& \left.+\frac{1}{\lambda_{2}} \sum_{k=M+L_{2}}^{n} x_{k}\left(1-\sum_{m=0}^{n-k} a_{m}^{2}\right) \mathbf{1}_{\left\{n \geq M+L_{2}\right\}}\right]
\end{aligned}
$$

where $E \triangleq \sum_{k=0}^{M+L_{1}-1} x_{k} T_{1}+\sum_{k=M+L_{1}}^{M+K-1} x_{k} T_{2}$. The performance measures can be obtained using Equations (17)-(19).

\section{Numerical Examples}

This section analyzes the performance of the LB scheme system and investigates its characteristics. Initially, the size of the EV waiting space is classified as either small, medium, or large sizes, with $K=5$ for small, $K=10$ for medium, and $K=30$ for large sizes. The operating method of the system is classified into three modes according to the battery-supply interval and the EVs' interarrival time. In Mode 1, the battery-supply interval is $T_{1}$ and EVs' interarrival time is $\lambda_{1}$. In Mode 3, these 
parameters are $T_{2}$ and $\lambda_{2}$, respectively. Mode 2 varies depending on the policy: The battery-supply interval is $T_{1}$ and the interarrival time of the EVs is $\lambda_{2}$ for the "AF" policy and $T_{2}$ and $\lambda_{1}$ for the "SF" policy. Numerical examples were created assuming the low, medium, and high levels, which were determined based upon $\lambda_{1} \times T_{1}$ for when the system started. However, because of the battery-supply interval and interarrival time of EVs change according to the control policy, the aforementioned offered load was unsuitable for expressing the overall characteristics of the system and was used only for simple classification. In Section 4.1 the performance of the system is examined under various conditions, and in Section 4.2 the cost structure is defined, and the economic system operation is investigated using cost analysis.

\subsection{System Performance}

In this section, we investigate the system performance using varying parameters. Initially, $\left(T_{1}, T_{2}\right)=(0.125,0.1)$ and $\left(\lambda_{1}, \lambda_{2}\right)=(3,1)$ were assumed for the low traffic circumstance, that is, the initial offered load is $\lambda_{1} \times T_{1}=0.375$. In the low loading situation, the congestion control is not necessary because EVs wait in the system. Table 1 shows the probability that the BSCS system operates in Mode 1 according to the combination of $\left(L_{1}, L_{2}\right)$ when $K=10$ and $M=3$. 'SF' and 'AF' denote the "Supply First" and "Arrival First" policy, respectively. In the case of low loading, the system is not congested, there are very few examples of exceeding the threshold $L_{1}$, and the probability of residing in Mode 1 is very high. The waiting time and EVs' queue size are negligible because their values are close to 0 . Furthermore, in this situation, these values are small and insignificant for a comparison of the two policies proposed in this study. When $M$ is larger than 3, there is almost no case of an EV waiting and the proportion of time during which the system remains in Modes 2 or 3 is zero. When the loading is low, the number of batteries in the battery queue exceeds $90 \%$ of its capacity. For every combination of $\left(L_{1}, L_{2}\right)$, the average number of batteries in the queue is approximately 2.7 when $M=3,6.7$ when $M=7$, and 9.7 when $M=10$. While, there are waiting EVs when $M=3$, the average is less than 0.02 and is negligible.

Table 1. Probabilities that the system is in Mode 1.

\begin{tabular}{ccc}
\hline \multirow{2}{*}{$\left.\boldsymbol{L}_{\mathbf{1}}, \boldsymbol{L}_{\mathbf{2}}\right)$} & \multicolumn{2}{c}{$\boldsymbol{P}$ (a BSCS is in Mode $\mathbf{1})$} \\
\cline { 2 - 3 } & SF Policy & AF Policy \\
\hline$(0,0)$ & 0.9927 & 0.9927 \\
$(0,2)$ & & \\
\hline$(0,4)$ & & \\
$(0,6)$ & 0.9922 & 0.9922 \\
$(0,8)$ & & \\
\hline$(2,2)$ & & \\
$(2,4)$ & 0.9998 & 0.9998 \\
$(2,6)$ & & \\
$(2,8)$ & & 1 \\
\hline$L_{1}, L_{2} \geq 4$ & 1 & \\
\hline
\end{tabular}

Next, the mid-size case of $K=10$ is examined. When $\left(T_{1}, T_{2}\right)=(0.167,0.125)$ and $\left(\lambda_{1}, \lambda_{2}\right)=$ $(3,1)$, the initial load $\lambda_{1} \times T_{1}$ is approximately 0.5 . In the case of mid-level traffic, the probability of EV blocking is close to zero as well. This means that the EVs are not blocked and almost all of them can receive service. Figure 2 shows the mean number of EVs $\left(L_{E V}\right)$ and batteries $\left(L_{B}\right)$ waiting according to the change of $\left(L_{1}, L_{2}\right)$. In mid traffic, the number of EVs is observed but the value is small. The mean EV queue length value is higher for the "SF" policy when $L_{1}=0$. When $L_{1}=0$, the "AF" policy operates with $\left(T_{1}, \lambda_{2}\right)$ and the "SF" policy operates with $\left(T_{2}, \lambda_{1}\right)$, meaning that controlling the interarrival time with high volatility is more effective than shortening the battery-supply interval. 


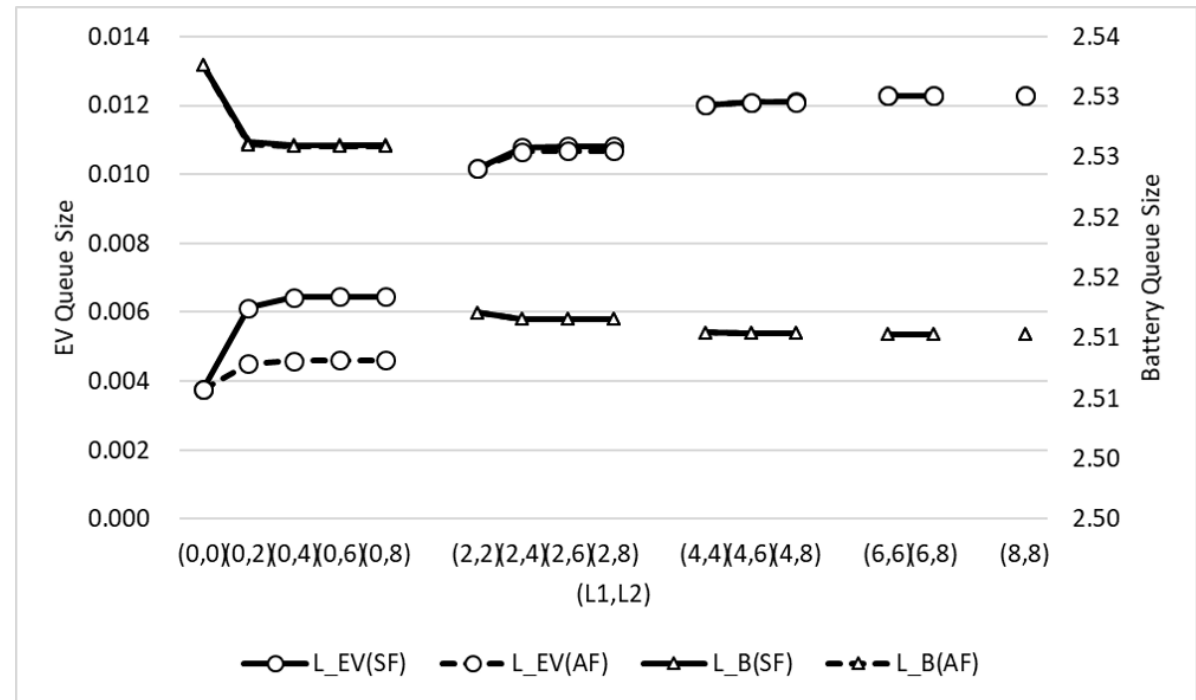

Figure 2. Electric vehicle (EV) and battery queue size $L_{E V}$ and $L_{B}$ of mid size queue for mid traffic.

Figure 3 shows a comparison of EV waiting time between the cases of $M=3$ and $M=7$ where $K=10,\left(T_{1}, T_{2}\right)=(0.125,0.1)$ and $\left(\lambda_{1}, \lambda_{2}\right)=(5,3)$. It is observed that, with an increase in $M$, there is a reduction in the EV waiting time. Evidently, this is because batteries can be immediately replaced if the number of completely charged and waiting batteries increases. The increase of the $M$ value implies an increase of in the EV processing capability; this is helpful for decreasing the mean EV queue length and the blocking probability. However, in a situation where the EV queue is completely filled, the queue length and the blocking probability cannot be drastically decreased regardless of the increase in the $M$ value; this is because a battery is installed in the $\mathrm{EV}$ as soon as it is supplied. As observed for the mean EV queue length, the "AF" policy shows relatively better performance when $L_{1}=0$. In the case of a large system with the mid-level traffic queue size of 30, EV blocking did not occur, and the EV queue size was zero. For the battery queue size, batteries were always added to exceed the capacity setup of $95 \%$ of $M$. When $M=10$ and $M=20$, on average 9.5 and 19.5 batteries were ready in the battery queue. While there were some differences in system performance between the two policies operating with the mid-sized traffic load, they were not significant.

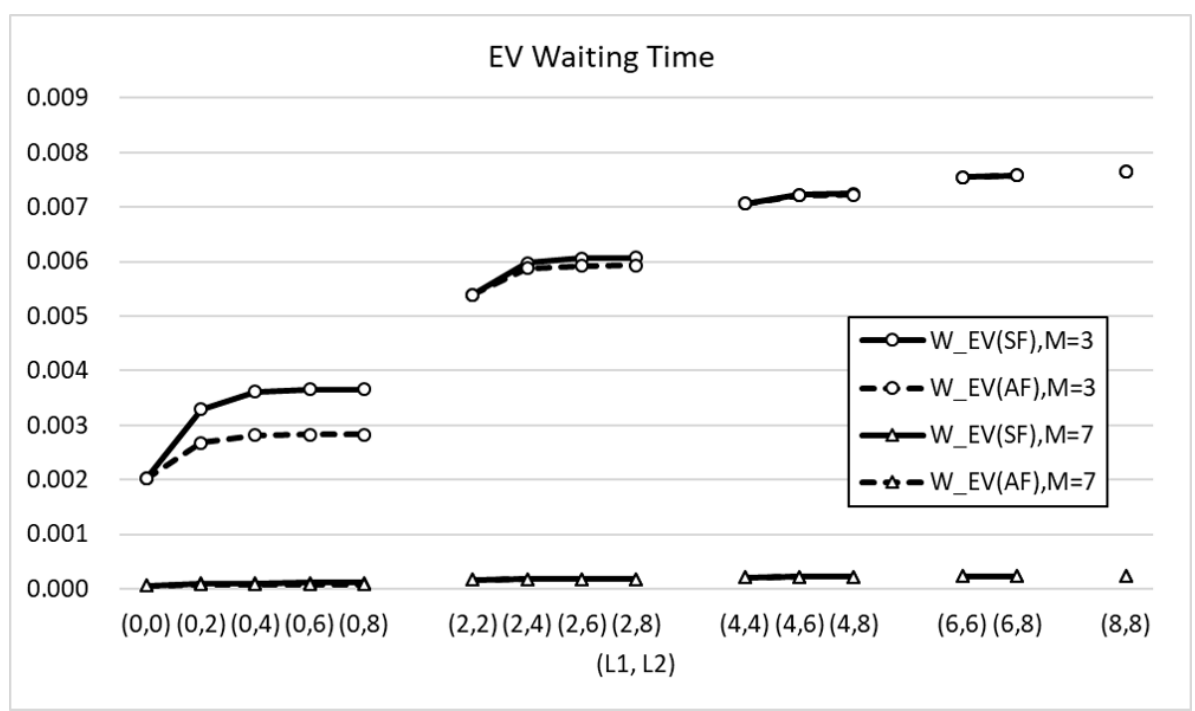

Figure 3. Waiting times of EVs $\left(W_{E V}\right)$ for two policies. 
The performance difference between the two policies occurs in a high traffic situation. For high traffic, we assumed cases in which the initial offered load $\lambda_{1} \times T_{1}$ is 0.8 or higher. Because this study considers a finite system, a situation in which $\lambda_{1} \times T_{1}>1$ can be assumed. Initially, Figure 4a shows the mean EV queue length, confirming that this value increases as the $L_{2}$ value increases for the same $L_{1}$ value. For Figure $4 a, M=10, K=3,\left(T_{1}, T_{2}\right)=(0.125,0.1)$, and $\left(\lambda_{1}, \lambda_{2}\right)=(7,5)$ are assumed. Furthermore, in the case of "SF" policy applied to a BSCS, when the volatility of EV-arrival is relatively high, it is confirmed that the number of waiting EVs is larger than that with the "AF" policy. Large $L_{1}$ or $L_{2}$ values imply that the congestion control policy is applied when the number of waiting EVs is large. This result, in which the initial load $\lambda_{1} \times T_{1}$ is 0.875 , is similar to the low and medium traffic cases. However, if the initial offered load is increased to 1.25 , where $M=10, K=7,\left(T_{1}, T_{2}\right)=(0.25,0.125)$ and $\left(\lambda_{1}, \lambda_{2}\right)=(7,5)$ are applied, the mean EV queue length is reversed: Figure $4 \mathrm{~b}$ shows that the queue length for the "AF" policy becomes larger than that seen with "SF" policy. This means that the effect of controlling the arrival interval is small if the EVs are severely queued up. Moreover, as a peculiar phenomenon, when the "AF" policy is applied first, the mean queue EV queue length increases more sharply than does that for the "SF" policy.

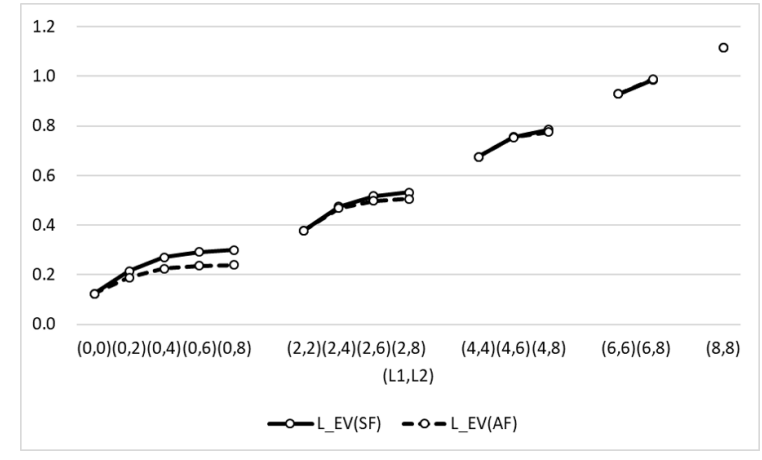

(a) Case of the initial load is 0.875

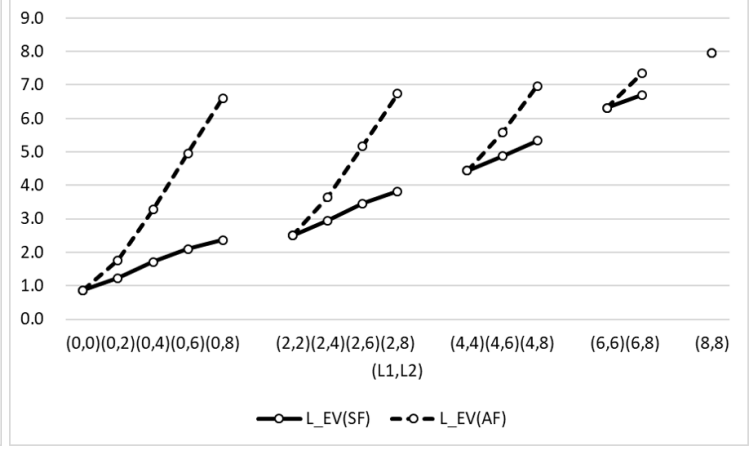

(b) Case of the initial load is 1.25

Figure 4. Mean EV queue length for high traffic cases.

Figure 5 shows the blocking probability for the case of $K=30, M=10,\left(T_{1}, T_{2}=0.25,0.125\right)$, and $\left(\lambda_{1}, \lambda_{2}\right)=(9,7)$. When $L_{1}$ and $L_{2}$ increase, it is confirmed that the increased range of blocking probability is larger with the "AF" policy as compared to the case of "SF" (where severe queued-up is observed).

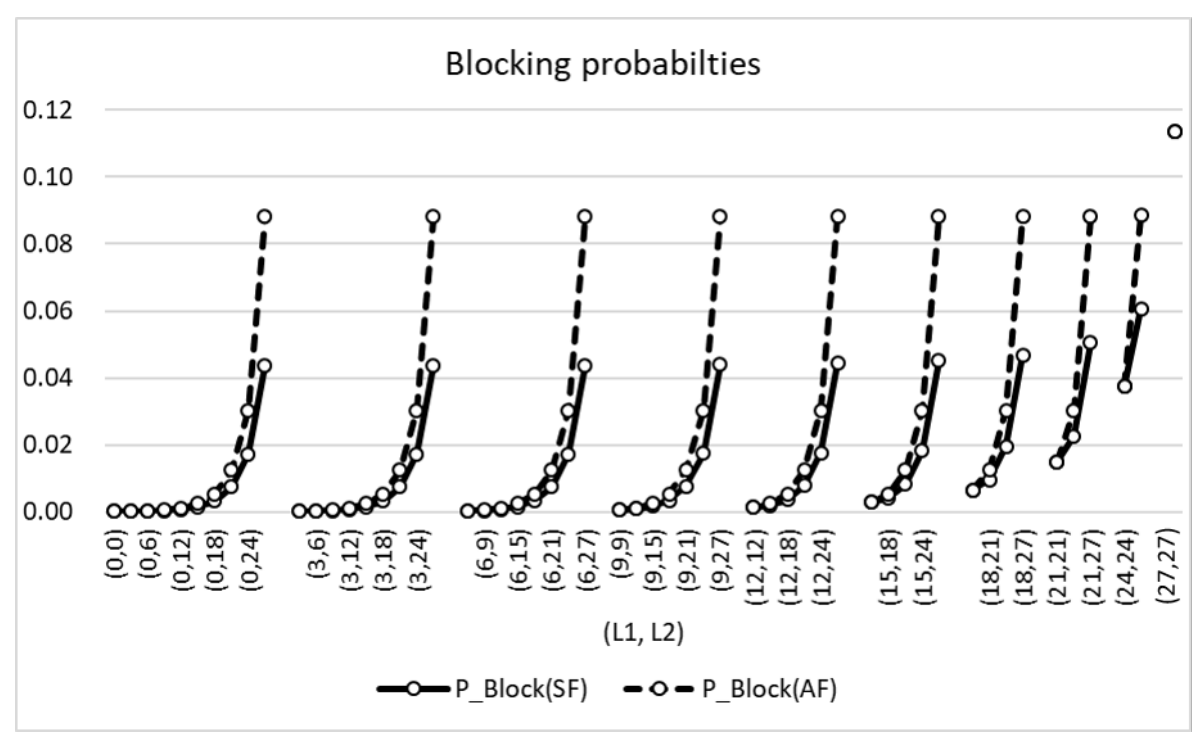

Figure 5. Blocking probabilities for two policies 


\subsection{Cost Analysis of the Operation of a Battery Swapping and Charging Station}

The economic system operation method is examined using cost analysis. Initially, the total cost per unit time for each policy is defined in Equation (23), and detailed terms are listed in Table 2. Blocking cost $P_{\text {Block }}$ refers to a cost that occurs when an EV leaves a BSCS without receiving service. While this is not an incurred cost, it is considered as an opportunity cost arising from losing a customer who is dissatisfied with the service. EV and battery holding costs, denoted by Cost Holding,EV and Cost $_{\text {Holding,Battery, }}$ are incurred when there is an EV or a battery in the system and this includes the costs of providing and maintaining the physical space. In particular, EV holding cost also includes the cost for customer dissatisfaction resulting from the EV waiting time, and it is assumed that this cost increases with an increase in waiting time. Note that we assume that the blocking and two types of holding costs are not dependent upon congestion control policies. The operating cost Cost $_{\text {Operating, } i}$ includes the maintenance cost, e.g., electricity and manpower, which allow the system to operate. A higher cost is likely incurred if a congestion control policy is applied by, for example, diminishing the battery-supply interval; this is because more resource (electrical power) is available. It is assumed that higher costs occur in the Mode 2 of the "SF" policy relative to those of the "AF" policy. This assumes that more resource is available for reducing the supply interval than for supporting bypassing efforts.

$$
\begin{aligned}
\text { Total } \operatorname{Cost}^{p}= & P_{\text {Block }}^{p} \times \operatorname{Cost}_{\text {Blocking }}+L_{E V}^{p} \times \operatorname{Cost}_{\text {Holding,EV }}+L_{B}^{p} \times \text { Cost }_{\text {Holding,Battery }} \\
& +\sum_{i=1}^{3} P^{p}(\text { BSCS is in Mode i }) \times \operatorname{Cost}_{\text {Operating, },}^{p}, \quad p \in\{A F, S F\} .
\end{aligned}
$$

Table 2. Cost structure in this study.

\begin{tabular}{cccccccc}
\hline \multirow{2}{*}{ Policy } & \multirow{2}{*}{ EV Blocking Cost } & \multicolumn{2}{c}{ Holding Cost } & \multicolumn{3}{c}{ Holding Cost } \\
\cline { 3 - 7 } & & EV & Battery & Mode 1 & Mode 2 & Mode 3 \\
\hline Supply First & 10 & 5 & 0.1 & 0.5 & 5 & 10 \\
\hline Arrival First & 10 & 5 & 0.1 & 0.5 & 2 & 10 \\
\hline
\end{tabular}

The total costs of the two policies are similar when loading is of low or medium level because the performance measures are similar. For a high loading case, we assume that $K=30, M=10,\left(T_{1}, T_{2}\right)=$ $(0.25,0.125)$ and $\left(\lambda_{1}, \lambda_{2}\right)=(7,5)$. Total costs are depicted in Figure $6 a$, which shows that total cost of the "AF" policy increases in accordance with the increase in the EV blocking probability $\left(P_{E V}\right)$ and is greater than that of "SF" due to the blocking cost. Moreover, the total cost of the "AF" policy increases sharply since the blocking probability increases rapidly as the $L_{2}$ value increases for the same $L_{1}$ value. It is observed that when medium loading is assumed $\left(K=10, M=3,\left(T_{1}, T_{2}\right)=(0.167,0.125)\right.$ and $\left.\left(\lambda_{1}, \lambda_{2}\right)=(3,1)\right)$, the total costs tend to decrease. This is because the probability that the BSCS is operating in Mode 1 increases. In other words, when the BSCS is not overly crowded, the length of the EV queue rarely exceeds the value $L_{1}$ and $L_{2}$ given that $L_{1}$ and $L_{2}$ are large. 


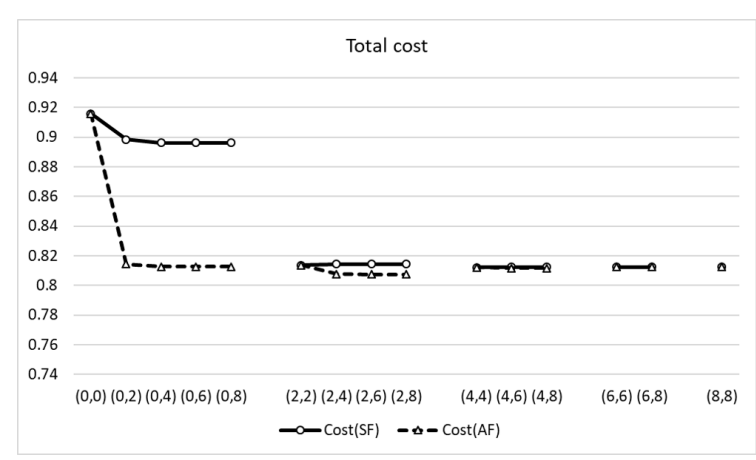

(a) System with medium loading

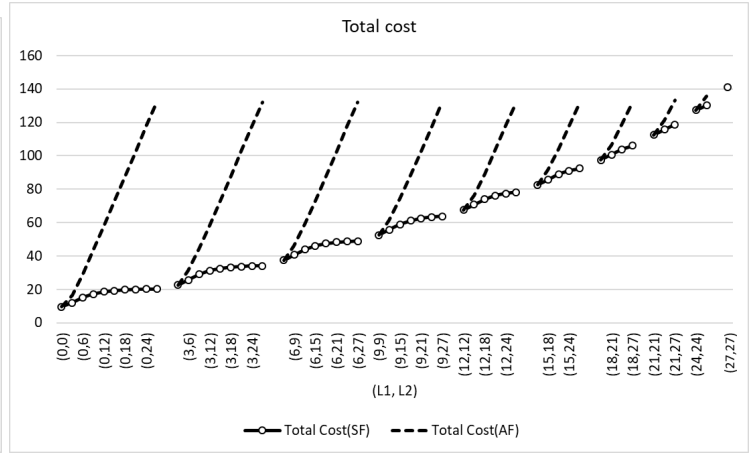

(b) System with high loading

Figure 6. Total cost for two policies.

\section{Concluding Remarks}

We analyzed a queueing model with a modified LB scheme and applied it to the operation of a BSCS. The modified LB scheme sets two thresholds in the EV queue size and employs a dynamic method to control the battery-supply intervals and EV-arrival intervals in response to the size of the packet queue. The (EV) queue length distributions at departure and at arbitrary times were derived using the embedded Markov chain and the supplementary variable technique. The performance measures discussed include the mean queue sizes for EVs and batteries and the blocking probabilities. Finally, numerical examples, including cost analysis were provided. It is observed that when the initial offered load is less than 0.9 , the "AF" policy outperforms (is more cost-effective than) the "SF" policy, but the difference is not overly significant. However, when controlling excessive arrivals failed, i.e., the initial offered load is far greater than 1 , the "SF" policy far surpasses the "AF" policy. This is because the cost for blocking EVs is relatively high in the proposed cost structure, and blocking probability increases sharply as $L_{2}$ increases when $L_{1}$ is fixed in the "AF" result.

Many limitations can be found when applying this study to the real situation. Among them, the most noticeable one is related to the assumption on the arrival of EVs. We assume EVs arrive at a charging station according to a Poisson process. While a Poisson process has the stationary increment property, non-stationary flow of vehicles is observed in real road traffic. Moreover, it is assumed that the types of batteries are homogeneous: Classes of EVs need to be classified unless it is a single kind. Finally, the volatility of the battery-supply interval can be also considered. Limitations of our model are expected to be overcome by postulating more realistic and detailed models which can be analyzed using simulation technique.

Author Contributions: This paper is the result of the joint work by two authors. D.I.C. designed the methodologies. D.-E.L. conducted the numerical experiment of the mathematical model. D.I.C. and D.-E.L. wrote the original draft. D.I.C. supervised all research processes. D.-E.L. reviewed and edited the final version of the manuscript. All authors have read and agreed to the published version of the manuscript.

Funding: This research was supported by Basic Science Research Program through the National Research Foundation of Korea(NRF) funded by the Ministry of Education(NRF-2017R1D1A3B03028784).

Conflicts of Interest: The authors declares no conflict of interest.

\section{References}

1. Gupta, V.; Kumar, R.; Panigrahi, B. Electric Vehicle Charging Management-Battery Charging vs. Swapping in Densely Populated Environments. Available online: https://smartgrid.ieee.org/newsletters/october2019/electric-vehicle-charging-management-battery-charging-vs-swapping-in-densely-populatedenvironments (accessed on 5 November 2019).

2. Varga, B.O.; Sagoian, A.; Mariasiu, F. Prediction of electric vehicle range: A comprehensive review of current issues and challenges. Energies 2019, 12, 946. [CrossRef] 
3. Adegbohun, F.; Von Jouanne, A.; Lee, K.Y. Autonomous battery swapping system and methodologies of electric vehicles. Energies 2019, 12, 667. [CrossRef]

4. Tillemann, L. The Faster, Cheaper, Better Way to Charge Electric Vehicles. Available online: https://www. wired.com/story/the-faster-cheaper-better-way-to-charge-electric-vehicles (accessed on 5 November 2019).

5. Forbes India. Battery Swapping: The Future of Electric Vehicle Charging. Available online: http://www. forbesindia.com/blog/technology/battery-swapping-the-future-of-electric-vehicle-charging (accessed on 5 November 2019).

6. Tan, X.; Sun, B.; Wu, Y.; Tsang, D.H. Asymptotic performance evaluation of battery swapping and charging station for electric vehicles. Perform. Eval. 2018, 119, 43-57. [CrossRef]

7. Turner, J. New directions in communications(or which way to the information age?). IEEE Commun. Mag. 1986, 24, 8-15. [CrossRef]

8. Tan, X.; Sun, B.; Tsang, D.H. Queueing network models for electric vehicle charging station with battery swapping. In Proceedings of the 2014 IEEE International Conference on Smart Grid Communications (SmartGridComm), Venice, Italy, 3-6 November 2014; pp. 1-6.

9. Lee, D.H.; Yang, W.S. The N-policy of a discrete time Geo/G/1 queue with disasters and its application to wireless sensor networks. Appl. Math. Model. 2013, 37, 9722-9731. [CrossRef]

10. De Cuypere, E.; De Turck, K.; Fiems, D. A queueing model of an energy harvesting sensor node with data buffering. Telecommun. Syst. 2018, 67, 281-295. [CrossRef]

11. Nicolaou, A.; Temene, N.; Sergiou, C.; Georgiou, C.; Vassiliou, V. Utilizing Mobile Nodes for Congestion Control in Wireless Sensor Networks. arXiv 2019, arXiv:1903.08989.

12. Jamian, J.; Mustafa, M.; Mokhlis, H.; Baharudin, M. Simulation study on optimal placement and sizing of battery switching station units using artificial bee colony algorithm. Int. J. Electr. Power Energy Syst. 2014, 55, 592-601. [CrossRef]

13. Raviv, T. The battery switching station scheduling problem. Oper. Res. Lett. 2012, 40, 546-550. [CrossRef]

14. Zou, B.; Xu, X.; De Koster, R. Evaluating battery charging and swapping strategies in a robotic mobile fulfillment system. Eur. J. Oper. Res. 2018, 267, 733-753. [CrossRef]

15. Nishimura, S.; Jiang, Y. An M/G/l vacation model with two service modes. Probab. Eng. Inf. Sci. 1995, 9, 355-374. [CrossRef]

16. Dudin, A. Optimal control for an $\mathrm{Mx} / \mathrm{G} / 1$ queue with two operation modes. Probab. Eng. Inf. Sci. 1997, 11, 255-265. [CrossRef]

17. Choi, D.I. MAP/G/1/K queue with multiple thresholds on buffer. Commun. Korean Math. Soc. 1999, 14, 611-625.

18. Dudin, A.N.; Nishimura, S. Optimal control for a BMAP/G/1 queue with two service modes. Math. Probl. Eng. 1999, 5, 255-273. [CrossRef]

19. Banik, A. Some aspects of stationary characteristics and optimal control of the BMAP/ G- G/ $1 / \mathrm{N}(\infty)$ oscillating queueing system. Appl. Stoch. Model. Bus. Ind. 2015, 31, 204-230. [CrossRef]

20. Gupta, U.; Samanta, S.; Goswami, V. Analysis of a discrete-time queue with load dependent service under discrete-time Markovian arrival process. J. Korean Stat. Soc. 2014, 43, 545-557. [CrossRef]

21. Li, H.; Zhu, Y. M (n)/G/1/N queues with generalized vacations. Comput. Oper. Res. 1997, 24, 301-316.

22. Li, H.; Zhu, Y.; Yang, P. Computational analysis of M (n)/G/1/N queues with setup time. Comput. Oper. Res. 1995, 22, 829-840. [CrossRef]

23. Choi, D.; Lim, D. Performance Analysis of Novel Overload Control with Threshold Mechanism. Math. Probl. Eng. 2016, 2016. [CrossRef]

24. Lee, J.; Un, C. Performance of dynamic rate leaky bucket algorithm. Electron. Lett. 1993, 29, 1560-1561. [CrossRef]

25. Choi, B.; Choi, D. Discrete-time analysis of the leaky bucket scheme with threshold-based token generation intervals. IEE Proc.-Commun. 1996, 143, 105-111. [CrossRef]

26. Choi, D.; Choi, B.; Sung, D. Performance analysis of priority leaky bucket scheme with queue-length-threshold scheduling policy. IEE Proc. Commun. 1998, 145, 395-401. [CrossRef]

27. Choi, D.; Lee, S. Performance analysis of the leaky bucket scheme with queue length dependent arrival rates. Bull. Korean Math. Soc. 2006, 43, 657. [CrossRef] 
28. Choi, D. Queueing analysis for traffic control with combined control of dynamic MMPP arrivals and token rates. J. Korea Soc. Ind. Appl. Math. 2013, 17, 103-113. [CrossRef]

29. Lee, T.T. M/G/1/N queue with vacation time and exhaustive service discipline. Oper. Res. 1984, 32, 774-784. [CrossRef]

(c) (P)

(C) 2020 by the authors. Licensee MDPI, Basel, Switzerland. This article is an open access article distributed under the terms and conditions of the Creative Commons Attribution (CC BY) license (http:/ / creativecommons.org/licenses/by/4.0/). 